\title{
Nonverbal Communication and the Effect
}

\author{
on Interpersonal Communication
}

\author{
Haiyan Wang \\ School of Foreign Languages, Qingdao University of Science and Technology \\ Qingdao 266061, China \\ E-mail: haiyanwang417@163.com
}

\begin{abstract}
It cannot be denied that the importance of interpersonal communication to the development of our society. Without interpersonal communication, people are unable to understand each other, cooperate with each other and promote the development of human society unceasingly. Therefore, the study of interpersonal communication has great significance.

Interpersonal communication is fulfilled through two forms: one is verbal behaviors, the other one is nonverbal behaviors. Verbal communication is the most common and significant form of communication. But it does not mean that we can ignore the importance of nonverbal communication .In interpersonal communication, many messages and meanings are expressed through touch, eye contact and gaze, slight diversity of tone, gesture and facial expressions with or without the help of verbal behaviors. We often try to understand one's heart thoroughly and make important judgment and decision to others according to nonverbal behaviors. So, the study of nonverbal communication and the effect on interpersonal communication has great practical significance.
\end{abstract}

Keywords: Nonverbal communication, Interpersonal communication, Nonverbal behavior

\section{Introduction}

The appearance and development of human society cannot be achieved without the communication of messages .As everyone knows, language is the main difference between animal and human beings. Without language, the appearance of human society is impossible. People communicate with each other through language, gestures, facial expressions and so on. Therefore people can understand each other better, improve interpersonal communication and finally promote the development of society.

From this angle, we can see the importance of studying interpersonal communication. Interpersonal communication has two forms: one is verbal communication, the other one is nonverbal communication. Verbal communication refers to the communication form which people communicate with each other by language or written language — characters. The way people transmit messages by the other methods (except language and characters) were called nonverbal communication. It cannot be denied that verbal communication is important, but at the same time the importance of nonverbal communication cannot be ignored. Famous scholar Larry A. Samovar once said: "most experts think that in face -to -face interpersonal communication, only about $35 \%$ of the "social meanings" is expressed by verbal behaviors, the rest is expressed by nonverbal communication." Therefore, studying nonverbal communication and the effect on interpersonal communication is very helpful to improve interpersonal communication.

\section{What is Nonverbal Communication?}

\subsection{The Definition of Nonverbal Communication}

For nonverbal communication, western scholars have provided us several definitions. According to Larry A. Samovar, "nonverbal communication involves all those nonverbal stimuli in a communication setting that are generated by both the source and his or her use of the environment and that have potential message value for the source or receiver."

The definition is consistent with current thinking in the field and reflects the cultural orientation, it can not only mark the boundaries of nonverbal communication, but also reflects how the process actually works. 


\subsection{The Classifications of Nonverbal Communication}

The earliest classification of nonverbal communication was issued by Ruesch and Kees according to the fundamental components of nonverbal communication. The classification is as follows:

- Sign language.

Including the gestures, which indicate meanings of words, numeral and punctuation marks. It not only includes each single gesture but also the whole sign language system.

- Action language.

Including various movements which can has different messages and meanings .For example, walking and drinking have two functions: one is to satisfy ourselves, the other one is to send a certain message to the partners.

- Object language.

Including various objects that are set up unintentionally and intentionally, such as tools, machine, work of art, construction structure, dress, etc.

M. Knapp put forward another classification through the study of relevant researches and works; all together there are seven categories: body motion and kinesic behavior, physical characteristics, touching behavior, paralanguage, proxemics, artifacts and environmental factors.

Afterwards, Jensen advanced another classification on the basis of the former scholars' study achievement on "silent language", which includes body motion and gestures, attitudes toward time, attitudes toward space and general habits in communication.

Condon John thought that nonverbal communication is relevant to psychology, sociology and physiology, its contents are extensive. Condon summarizes twenty-four types of nonverbal behaviors, which already drew scholars' attention; Including: gestures, facial expressions, posture, costume and hair style, walking posture, proxemics, touching behavior, eye gaze and contact, architectural design and home decoration, signs and symbols, body odor, paralanguage, color, make-up, conception about time, silence, etc.

\section{Nonverbal Communication's Effect on Interpersonal Communication}

As everybody knows, nonverbal communication is the indispensable part of interpersonal communication. Whether the use of nonverbal communication is proper or not decides the result of interpersonal communication directly . It is better to choose several common and representative nonverbal behaviors to expound how nonverbal communication affects interpersonal communication under the influence of various limiting factors, especially cultural differences.

\subsection{Body Behavior}

Body behavior refers to the expression and movement which transfer communicative messages. For the study of body behavior , we can trace back to Aristotle - the most great Greece philosopher . The first man to study body behavior formally is Darwin, he point out that there were many similar expression between animal and human beings in his book The Expression of the Emotion in Man and Animals, (1872). And the most successful scholar who systematizes body behavior is Rag L. Birdwhistell, he advanced the theory of kinesics in his work Introduction to Kinesics, (1952). Fast, Body Language, (1970), is another summary and representative work in this field. Since then, body behavior became a new bi-disciplinary science, and continuously there are new study achievements emerge.

According to estimation, people can do up to 270,000 types of gestures and movement .The meanings of so many gestures and movement are complicated. Some of the meanings are definite, some are vague; some are used in communication, some are only self-expression; some express emotion, some reflect personality and attitude .For this reason, the classification of body behavior is complex.

In the study field of intercultural communication, the most common classification of body behavior is put forward by Samovar. He divided body behavior into: general appearance and dress, body movement, posture, gesture, facial expressions, eye contact and gaze, touch, smell, paralanguage, etc. The following part will expound body behavior according to Samovar's classification.

\subsubsection{General Appearance and Dress}

Concern with how one appears is universal .As far back as the Upper Paleolithic period (about forty thousand years ago), our ancestors were using bone for necklaces and other bodily ornaments. From that period to the present, historical and archaeological evidence has shown that people are fixated on their bodies. In modern society, people usually make judgment based on personal appearance, dress, and the objects we carry around or place on our bodies. When deciding whether or not to strike up a conversation with a total stranger, people are influenced by the way that person looks .If the person's dress is neat and facial expression is mild, people will be willing to talk to him or her. A charming girl in suit may get a new job more easily. A rich man may wear a diamond ring or smoking cigar to reveal 
that he is wealthy and superior. These may make the person who wants to talk to the man feel depressing, humble or annoying. It can be called appropriate that people dress up themselves according to their age, class, occupation and so on. For instance, police and attendant should wear uniforms; merchant and government officials 'dress are exquisite; artists' dress should be fashionable; students 'dress style should be leisurable, etc. Just as Ruben says, "we make inferences about another's intelligence, gender, age, approachability, financial well-being, class, tastes, values, and cultural background from attractiveness dress, and personal artifacts." Our culture's obsession with attractiveness is so deep-seated, and begins so early in life, that, as one study revealed, even very young children select attractive friends over less attractive ones.

In all, it is natural people make judgment from a person's general appearance and dress, if a person's general appearance and dress are not proper, it may cause misunderstanding or even some worse consequences. Therefore, it is necessary to learn how to make our general appearance and dress more proper in practice, thus we can communicate efficaciously.

\subsubsection{Body Movement}

People have always known that action communicates .The study of how movement communicates is called kinesics. In general, kinesic cues are those visible body shifts and movements that can send messages about (1) our attitude toward the other person, e.g., standing face-to-face with a friend, or leaning forward may show that we are relaxed. (2) Our emotional state, e. g, tapping on the table or playing with coins can mean we are nervous, and (3) our desire to control our environment, e. g, motioning someone to come closer means we want to talk to him or her.

Scholars have suggested that we can make as many as 700,000 distinct physical signs, any attempt at cataloging them would be frustrating and fruitless. Moreover, the ways of how to use and interpret these movements will be different if they belong to different cultures, and in most instances the messages the body generates operate only in combination with other messages.

\subsubsection{Posture}

The same as body movement, posture can communicate varied messages too. Posture offers insight into a culture's deep structure. In many Asian cultures, the bow is much more than a greeting, which signifies that culture's concern with status and rank. In Japan, for example, low posture is an indicator of respect. Although it appears simple to the outsider, the bowing ritual is actually rather complicated. The person who occupies the lower station begins the bow, and his or her bow must be deeper than the other person's. The superior, on the other hand, determines when the bowing is to end. When the participants are of equal rank, they begin the bow in the same manner and end at the same time .The Thai people use a similar movement called the wai. The wai movement - which is made by pressing both hands close together in front of one's body, with the fingertips reaching to about neck level — is used to show respect .The lower the head comes to the hands, the more respect is shown.

In the United States people are not accustomed to squat, and view this posture unrefined. If it is possible, they prefer kneel or sit on ground. Provided they really have to squat, they often squat with one tip of toe and the other sole of foot place on ground. Women avoid squat especially when they are in skirts, if it can not be avoid, they would use their skirt to cover their knees to maintain elegance, not like some Chinese women prefer place the skirt between legs.

People communicate all the time. In interpersonal communication, under certain circumstances, postures have different meanings. Use and understand them correctly is helpful to the harmony among people, this is also the aim of communication.

\subsection{Space and Distance}

The flow and shift of distance between us and the people with whom we interact are as much a part of communication experiences as the words we exchange. We use space and distance to convey meanings. The study of space and distance called proxemics, the word is created by Edward T. Hall .In his book The Silent Language, (1959), he divided the distance which people keep in interpersonal communication into eight sections:

\section{Insert Table 1 Here}

The nearer the more secret the messages people transmit are; the further the louder the volumes of people are.

Afterwards, people divide the distance people keep in conversation into four zones:

1) Intimate Zone. (0-18 feet)

From the angle of psychology, this zone belongs to ourselves, except close people, the others who rush in the zone are unwelcome. People in this zone often talk about top-secret things. But in crowded public place, such as on a bus or in a theater, the intrusion of intimate zone cannot be avoided.

2) Personal Zone. (1.5-4 inches)

This is the zone for close friends, the contents of conversation are generally about private affairs, and the speaker talks 
softly .Of course, if people are in outdoors, the volume will be higher.

3) Social Zone. (4-12 inches)

It is the normal distance of social interaction .In this zone people usually talk about public issue, which are not necessary to keep secret to other people, the volume of speaker is also moderate.

4) Public Zone. (over 12 inches)

In this zone, the speaker's volume is quite high, commonly it happens in the situation of lecture or play act.

There are also gender differences in the use of space .Men use space as a means of asserting their dominance over women, as in the following: one, they claim more personal space than women; two, they more actively defend violations of their territories which are usually much larger than the territories of women; three, under conditions of high density, they become more aggressive in their attempts to regain a desired measure of privacy; and the last one is, men more frequently walk in front of their female partner than vice versa .

\subsection{Silence}

An African proverb states, "Silence is also speech." We contend that silence sends us nonverbal cues concerning the communication situations in which we participate. Silent cues affect interpersonal communication by providing an interval in an ongoing interaction during which the participants have time to think, check or suppress an emotion, encode a lengthy response, or inaugurate another line of thought. Silence also helps provide feedback, informing both sender and receiver about the clarity of an idea or its significance in the overall interpersonal exchange. Silence cues may be interpreted as evidence of agreement, lack of interest, injured feelings, or contempt. Like olfactory and tactile cues, silence cues transcend the verbal channel, often revealing what speech conceals. The intercultural implications of silence are as diverse as those of other nonverbal cues, a particular instance of silence is: in response to the question "Will you marry me?", silence in English would be interpreted as uncertainty; in Japanese it would be interpreted as acceptance. In Igbo, it would be considered a denial if the woman were to continue to stand there, and an acceptance if she ran away.

\subsection{Signs and Symbols}

Signs and symbols are a type of nonverbal communication forms which can represent verbal communication. In modern society, people often feel unsatisfied with or unable to use language as communication method, so we often seek the help of various signs and symbols which can directly stimulate people's sense organ, and use these signs and symbols to substitute language so that we can make response more directly, effectively and rapidly.

Signs and symbols have two forms: symbolic signs and symbols and notional signs and symbols. Symbolic signs and symbols represent abstract meanings, such as the crucifix represents Christianity. All symbolic signs and symbols are crystallizations of cultures; they have deep historical tradition and contain plentiful emotions. Notional signs and symbols can express certain meanings more succinctly and directly. Initially people use these signs and symbols on traffic routes, such as the road sign (highway), signal banner and lamp signal (ocean). At present, they are used in many fields and occasions. As the life rhyme of modern society accelerating unceasingly, in diverse public places, it is common that use signs and symbols to substitute written explanation. Along with the rapid development of international communication, the emergence of generally used signs and symbols eliminate language barrier and accepted by people universally.

\section{Conclusion}

It is evident that nonverbal communication is important to interpersonal communication. Now we all know it is impossible to list all the nonverbal behaviors, for nonverbal communication is so complicated. Because of the differences in gender, occupation, class, race, culture and so on, the messages and meanings of nonverbal behaviors become more complex. Especially the effect of culture, in different cultures, the same nonverbal behavior even transmits just opposite messages and meanings. Therefore, the study of intercultural communication is imperative, it would be helpful to the harmony among countries.

The study on nonverbal communication has great effect of guidance on practice. Through the study on nonverbal communication, we know what and how we should do in certain communication circumstances, thus we can improve our nonverbal behaviors. In recent years, the use of nonverbal communication methods has new development. For instance, as the rapid development of Internet, now it is common to chat with another person on Internet by chat software, such as QQ, MSN Messenger, UC, etc .If we want to tell the person we are happy, we can send an emotion picture with a smiling face, and then the person will understand your mood. This way is more interesting and vivid than characters.

In all, as long as we can comprehend nonverbal communication comprehensively and understand the different messages and meanings of various nonverbal behaviors under the effect of different factors, it is not hard to behave properly and 
make correct judgment to other people's nonverbal behaviors, thus the aim of improving interpersonal communication can be reached. In the future, we have reasons to believe that nonverbal communication will get inconceivable new development, bring us more surprises and change the traditional communication patterns more greatly.

\section{References}

B. D. Ruben. (1992). Communication and Human Behavior (Third Edition). New Jersey: Englewood Cliffs, 213.

E. T. Hall. (1959). The Silent Language. New York: Fawcett.

Julius Fast. (1988). Body Language. New York: Pocket Books.

Larry A. Samovar, Richard E. Porter \& Lisa A. Stefani. (2000). Communication between Cultures (Third Edition). California: Wadsworth publishing company.

Larry A. Samovar. (1981). Understanding Intercultural Communication. California: Wadsworth publishing company.

P. Ekman, R. Sorenson \& W. V. Friesen. (1969). Pan-Cultural Elements in Facial Display of Emotion., Science, 64, 86-88.

P. Ekman. (1975). Face Muscles Talk Every Language. Psychology Today, New York: September, 35-39.

Rag L. Birdwhistell. (1952). Introduction to Kinesics. Kentucky: Univ. of Louisville Press.

Table 1.

\begin{tabular}{|l|l|l|}
\hline 1. & Very close & $3-6$ feet \\
\hline 2. & Close & $8-12$ feet \\
\hline 3. & Near & $12-20$ feet \\
\hline 4. & Neutral & $20-36$ feet \\
\hline 5. & Neutral & $4.5-5$ inches \\
\hline 6. & Public distance & $5.5-8$ inches \\
\hline 7. & Across the room & $8-20$ inches \\
\hline 8. & Stretching the limits of distance & $\begin{array}{l}20-24 \text { inches (indoors) } \\
\text { up to } 100 \text { inches (outdoors) }\end{array}$ \\
\hline
\end{tabular}

